\title{
Childhood trauma in adults with social anxiety disorder and panic disorder: a cross-national study
}

\author{
C Lochner', S Seedat', C Allgulander ${ }^{2}$, M Kidd ${ }^{3}$, D Stein ${ }^{1,4}$, A Gerdner ${ }^{5}$ \\ ${ }^{1}$ MRC Unit on Anxiety and Stress Disorders, Department of Psychiatry, University of Stellenbosch, South Africa \\ 2Department of Clinical Neuroscience, Karolinska Institutet, Sweden \\ ${ }^{3}$ Centre for Statistical Consultation, Department of Statistics \& Actuarial science, University of Stellenbosch, South Africa \\ ${ }^{4}$ Department of Psychiatry, University of Cape Town, South Africa \\ ${ }^{5}$ Department of Social Work, Mid Sweden University, Östersund, Sweden
}

\begin{abstract}
Objectives: The influence of childhood trauma as a specific environmental factor on the development of adult psychopathology is far from being elucidated. As part of a collaborative project between research groups from South Africa (SA) and Sweden focusing on genetic and environmental factors contributing to anxiety disorders, this study specifically investigated rates of childhood trauma in South African and Swedish patients respectively, and whether, in the sample as a whole, different traumatic experiences in childhood are predictive of social anxiety (SAD) or panic disorder (PD) in adulthood. Method: Participants with SAD or PD (85 from SA, 135 from Sweden) completed the Childhood Trauma Questionnaire (CTQ). Logistic regression was performed with data from the two countries separately, and from the sample as a whole, with primary diagnoses as dependent variables, gender, age, and country as covariates, and the CTQ subscale totals as independent variables. The study also investigated the internal consistency (Cronbach alpha) of the CTQ subscales. Results: SA patients showed higher levels of childhood trauma than Swedish patients. When data from both countries were combined, SAD patients reported higher rates of childhood emotional abuse compared to those with PD. Moreover, emotional abuse in childhood was found to play a predictive role in SAD/PD in adulthood in the Swedish and the combined samples, and the same trend was found in the SA sample. The psychometric qualities of the CTQ subscales were adequate, with the exception of the physical neglect subscale. Conclusion: Our findings suggest that anxiety disorder patients may differ across countries in terms of childhood trauma. Certain forms of childhood abuse may contribute specific vulnerability to different types of psychopathology. Longitudinal studies should focus on the potential sequential development of SAD/PD among individuals with childhood emotional abuse.
\end{abstract}

Keywords: Childhood trauma; Social anxiety disorder; Panic disorder; Cross-national

Received: 26-05-2009

Accepted: 13-10-2009

\section{Introduction}

The association between retrospectively reported childhood adverse experiences and psychiatric morbidity in adulthood is documented in several North American and European surveys of community and clinical samples. ${ }^{1}$ For example, adverse parental rearing styles (including lack of care and overprotection) have been associated with the risk for anxiety disorders. ${ }^{2}$

Although some theorists have suggested that childhood

Correspondence

C Lochner

PO Box 19063, Tygerberg, 7505, South Africa

email: cl2@sun.ac.za abuse is a relatively non-specific risk factor for

psychopathology in adulthood ${ }^{3}$, others have hypothesized that certain forms of childhood abuse may contribute specific vulnerability to different types of psychopathology. Although little research has examined the relative specificity of various forms of abuse to different anxiety disorders, a few studies have to some extent investigated forms of childhood abuse in panic disorder (PD) and social anxiety disorder (SAD). For example, one case-control study found higher rates of childhood trauma in patients with $\mathrm{PD}^{4}$, while a German study of antecedents of SAD reported that traumatic childhood experiences, including separation from parents, parents' marital problems, sexual abuse in the family, and familial violence, were significantly more common in patients with $\mathrm{SAD}$ 
than in controls. ${ }^{5}$ In another study by the same group an association was found between early traumatic life events and $\mathrm{PD} .{ }^{6}$ Negative interpersonal life events were also reported in a Swedish study of patients with SAD. ${ }^{7}$

A few studies have focused on the role of the various forms of childhood adversity in adult morbidity. Specifically, childhood emotional abuse, more so than physical or sexual abuse, has been associated with diagnoses of major depression and SAD. ${ }^{9}$ The various types of emotional abuse that have been implicated in the development of social anxiety and $\mathrm{SAD}$ include excessive teasing, criticism, bullying, rejection, ridicule, humiliation, and exclusion by significant others. ${ }^{9-12}$ An association between emotional abuse and neglect in childhood and adult PD has also been suggested. ${ }^{13,14}$ Neglect may also play a role in SAD; for example, in a community study, Chartier et $\mathrm{al}^{15}$ reported that, amongst other things, a lack of close personal relationships with adults was significantly associated with a diagnosis of $\mathrm{SAD}$ in young adulthood. Stein and colleagues found higher rates of retrospectively recalled childhood physical and sexual abuse in patients with SAD, PD and obsessive-compulsive disorder than in controls. ${ }^{16}$ Of note, Safren et al. found significantly lower rates of recalled childhood physical or sexual abuse in patients with PD than in patients with SAD. ${ }^{17}$ In contrast, a study by Mancini et al. found no difference in reported sexual abuse across all of the anxiety disorders that were investigated. ${ }^{18}$ Similarly, neither the number nor the types of past traumatic events (which included assault, rape, witnessing injury or death, and natural or man-made disasters), were found to differentiate patients with PD from those with other anxiety disorders in a study by Hofmann et al. Findings on the possible associations between various forms of recalled childhood maltreatment and subsequent anxiety disorders specifically, have been inconclusive. ${ }^{19}$

Rates of childhood adversity, and subsequent psychiatric morbidity in adulthood, may vary across different environments. It has been suggested that if environmental factors such as violence, crime and poverty are major mental health determinants, then it is plausible that in those settings (i.e. low-income countries) with high levels of these environmental factors, the mental health of individuals may be compromised. We undertook this exploratory study to determine whether patients with anxiety disorders differ across two countries in terms of childhood trauma history one a high-income developed, and the other a lower income (i.e. developing) country. As part of a Swedish-South African collaborative project, we specifically investigated whether childhood trauma history is predictive of adult SAD or PD in South African and Swedish patients, respectively. Given our use of data obtained with the Childhood Trauma Questionnaire $(\mathrm{CTQ})^{20}$, we also investigated the internal consistency (Cronbach alpha) of the CTQ subscales.

\section{Method}

\section{Subjects}

Eighty-five (n=85) South African and 135 Swedish patients were included. The study was conducted on outpatients in 5 provinces of South Africa and in Stockholm, Sweden. In South Africa, patients were recruited by advertisements in the media and recruitment calls to psychiatrists and psychologists, primary care practitioners, and advocacy groups, and in
Sweden they were recruited by advertisement and a telephone interview. All patients met DSM-IV criteria for a primary lifetime diagnosis of SAD or PD with/-out agoraphobia. Diagnostic status was assessed with the Structured Clinical Interview for the Diagnosis of Axis I Disorders - Patient Version (SCID-I/P) ${ }^{21}$ in the South African sample, and with the Mini International Neuropsychiatric Interview (MINI) in the Stockholm sample. Concordance between the SCID and MINI was found to be good for both $\mathrm{SAD}$ and PD. ${ }^{22}$ Patients with other primary psychiatric conditions, including psychosis and substance use disorders, as well as inadequate understanding of the aims and practical implications of participation, and unwilling to provide consent after reading the information and consent forms, were excluded. The study was approved by the institutional review boards of the University of Stellenbosch in SA and the Karolinska Institutet in Sweden.

\section{Self-reports}

The 28-item $\mathrm{CTQ}^{20}$ was used to assess the frequency and severity of different types of recalled childhood interpersonal trauma. The items are divided into 5 subscales: emotional abuse, physical abuse, sexual abuse, emotional neglect and physical neglect. The CTQ also includes a 3-item Minimization/Denial (MD) scale, which is indicative of potential underreporting of maltreatment or idealizing the family of origin. The scoring of responses on the 3 items comprising the MD scale were dichotomized ("very often true" = 1, all other responses $=0$ ) and summed. A total of 1 or greater suggests the possible underreporting of maltreatment (false negatives). ${ }^{23}$ A score of 3 indicates extreme minimization or denial of maltreatment during childhood.

\section{Data analysis}

Demographic and CTQ data were compared between the sites with chi-square and t-tests as appropriate. A three-way ANOVA was used to assess the combined and individual associations of diagnosis, country and gender with CTQ scores, using Bonferroni corrections. Following separate country analyses, logistic regression was performed on the combined South African and Swedish datasets to examine, in this larger sample, whether childhood trauma history is predictive of adult $\mathrm{SAD}$ or $\mathrm{PD}$, with primary diagnosis as the dependent variable, gender, age, and country as covariates and CTQ (sub-) scale totals as the independent variables. Due to the ordinal nature of the MD-scores, non-parametric bootstrap confidence intervals were calculated and statistical significance determined from these bootstrap results. Regression analyses were repeated after cases with extreme scores on the MD scale were excluded. The internal consistency (Cronbach's alpha) of the CTQ was also assessed. Spearman rank order correlations were used to determine univariate relationships between ordinal variables. P-values for the Wald chi-square statistics were reported. P-values $<.05$ were considered as significant.

\section{Results}

\section{Demographic characteristics:}

Eighty-five South African patients (36 male; 49 female) and 135 Swedish patients (65 male, 70 female) were included. The South African sample comprised of 48 SAD and 37 PD 
Table I: Demographics by diagnosis, age and country

\begin{tabular}{|c|c|c|c|c|c|c|c|}
\hline & \multicolumn{3}{|c|}{$\begin{array}{c}\text { South Africa } \\
N=85,36 \text { Male; } 49 \text { Female }\end{array}$} & \multicolumn{3}{|c|}{$\begin{array}{c}\text { Sweden } \\
N=135,65 \text { Male; } 70 \text { Female }\end{array}$} & \multirow[t]{2}{*}{$\begin{array}{c}\text { Statistics } \\
\text { (South Africa Vs. Sweden*) }\end{array}$} \\
\hline & Total Sample & Male & Female & Total Sample & Male & Female & \\
\hline $\begin{array}{l}\text { Panic Disorder, N (\%) } \\
\text { Social Anxiety Disorder, N (\%) } \\
\text { Age, Years (Sd) }\end{array}$ & $\begin{array}{l}37 \\
48 \\
37.8(12.8)\end{array}$ & $\begin{array}{l}9(24.32 \%) \\
27(56.25 \%) \\
38.33(12.59) \ngtr\end{array}$ & \begin{tabular}{|l|}
$28(75.68 \%)$ \\
$21(43.75 \%)$ \\
$37.35(13.11)^{\star \star}$
\end{tabular} & $\begin{array}{l}52 \\
83 \\
46.4(11.2)\end{array}$ & $\begin{array}{l}22(42.31 \%) \\
43(51.81 \%) \\
46.14(10.99)^{\ngtr}\end{array}$ & $\begin{array}{l}30(57.69 \%) \\
40(48.19 \%) \\
46.6(11.45)^{\star \star}\end{array}$ & $\begin{array}{l}\chi^{2}=0.707, P=0.4 \\
t=-5.09, p<0.001\end{array}$ \\
\hline
\end{tabular}

patients, while the Swedish sample comprised $83 \mathrm{SAD}$ and 52 PD patients. The gender distribution was similar at both sites; in the randomly recruited combined patient samples from both South Africa and Sweden there were more males than females with $\mathrm{SAD}$, and more females with PD compared to males. Swedish patients were older at the time of their assessment than South African patients ( $\mathrm{t}=-5.09, \mathrm{p}<0.001$ ) (Table I).

South African patients reported significantly more childhood trauma than patients from Sweden. In particular, their rates of sexual abuse, emotional and physical neglect were significantly higher than those of Swedish patients. Regarding emotional and physical abuse, country differences were not statistically significant but were in the same direction (i.e. higher in South African patients) as with the other types of childhood trauma (Table II).

Findings from subsequent analyses also reflected similarities in the two samples. In the combined sample, women reported significantly higher rates of emotional abuse $(\mathrm{p}=0.02)$ and sexual abuse $(\mathrm{p}<0.01)$ than men. When diagnostic groups were separated (combined sample), ANOVA showed significantly higher rates of emotional abuse in patients with SAD compared to patients with PD $(p<0.01)$ (Table II; Figure 1). More specifically, SAD was associated with higher emotional abuse scores while PD was associated with lower emotional abuse scores.

Logistic regression analysis - with primary diagnosis as the dependent variable, and gender, age, and country as covariates, and CTQ sub-scale totals as independent variables - also suggested a predictive association between emotional
Figure 1: Rates of emotional abuse in the combined sample (SA + Sweden): Social anxiety disorder vs. Panic disorder

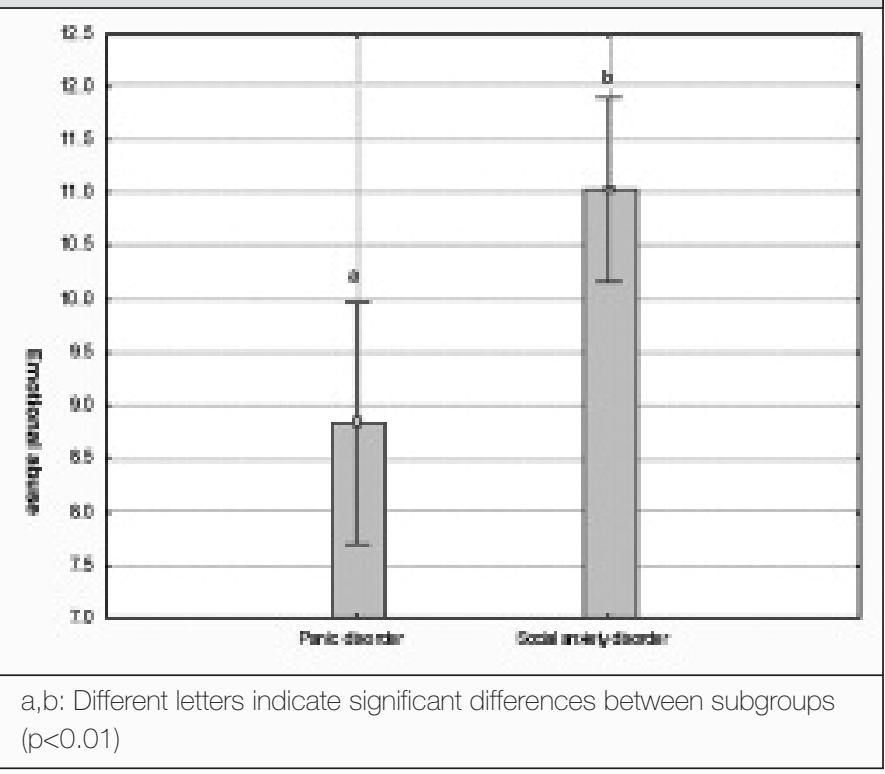

abuse and SAD or PD ( $p=0.003)$. This association remained unaltered after exclusion of patients with extreme scores on the MD-scale.

A three-way factorial ANOVA was subsequently performed to investigate the combined and individual relationships between diagnosis, country and gender, and the CTQ

\begin{tabular}{|c|c|c|c|c|c|c|c|c|c|}
\hline \multirow[t]{2}{*}{ Ctq-Subscale } & \multicolumn{3}{|c|}{$\begin{array}{c}\text { Gender } \\
\text { (SA and Sweden Combined) }\end{array}$} & \multicolumn{3}{|c|}{ Country } & \multicolumn{3}{|c|}{$\begin{array}{c}\text { Diagnosis } \\
\text { (SA and Sweden Combined) }\end{array}$} \\
\hline & Male & Female & P-Value & South Africa & Sweden & P-Value & Panic Disorder & $\begin{array}{l}\text { Social Anxiety } \\
\text { Disorderd }\end{array}$ & P-Value \\
\hline $\begin{array}{l}\text { Emotional Abuse } \\
\text { Physical Abuse } \\
\text { Sexual Abuse } \\
\text { Emotional Neglect } \\
\text { Physical Neglect } \\
\text { Ctq-Total }\end{array}$ & \begin{tabular}{|l|}
$9.5(4.7)$ \\
$6.9(3.1)$ \\
$5.3(1.4)$ \\
$14.9(5.5)$ \\
$9.7(3.4)$ \\
$46.3(12.1)$
\end{tabular} & \begin{tabular}{|l}
$10.6(5.2)$ \\
$7.0(3.4)$ \\
$6.9(4.1)$ \\
$15.2(5.9)$ \\
$10.0(3.4)$ \\
$49.6(14.1)$
\end{tabular} & $\begin{array}{l}0.02 \\
0.83 \\
<0.01 \\
0.77 \\
0.97 \\
0.07\end{array}$ & \begin{tabular}{|l|}
$10.9(5.4)$ \\
$7.3(3.4)$ \\
$7.1(4.5)$ \\
$17.9(5.4)$ \\
$12.6(1.7)$ \\
$55.8(8.6)$
\end{tabular} & \begin{tabular}{|l|}
$9.6(4.6)$ \\
$6.7(3.2)$ \\
$5.6(1.2)$ \\
$13.3(5.1)$ \\
$8.2(3.0)$ \\
$43.2(13.4)$
\end{tabular} & $\begin{array}{l}0.14 \\
0.22 \\
<0.01 \\
<0.01 \\
<0.01 \\
<0.01\end{array}$ & $\begin{array}{l}9.1(4.4) \\
6.7(3.5) \\
6.3(3.7) \\
15.3(5.9) \\
10.2(3.5) \\
47.7(13.2)\end{array}$ & \begin{tabular}{|l|}
$10.7(5.2)$ \\
$6.9(3.1)$ \\
$6.1(3.0)$ \\
$14.9(5.5)$ \\
$9.7(3.3)$ \\
$48.3(13.3)$
\end{tabular} & $\begin{array}{l}<0.01 \\
0.69 \\
0.48 \\
0.47 \\
0.43 \\
0.27\end{array}$ \\
\hline
\end{tabular}


subscale scores. No significant interactions were found for emotional abuse (Table III) or any of the other trauma subscales.

The only exception was in the case of MD scores where there was a significant diagnosis/country interaction, indicating that differences in MD scores between the two diagnoses were dependent on the country regardless of gender. Among PD patients in particular, South Africans had significantly higher MD-scores $(p<0.01)$ than their Swedish counterparts whereas in the $\mathrm{SAD}$ group, there were no country differences in MD scores ( $p=1.00$ ) (Table IV; Figure 2). (Notably, since MD scores were ordinal and varied discretely from 0 to 3, non-parametric bootstrap confidence intervals were calculated and statistical significance was determined from these bootstrap results.)

In addition, in terms of the MD subscale, the scoring of responses on the 3 items comprising the scale were also dichotomized (see Methods - Self-reports). Chi square analyses indicated that an increased number of PD patients from South Africa compared to Swedish PD patients possibly underreported maltreatment as indicated by an MD total score of 1 or greater $\left(\chi^{2}=3.765, p=0.052\right)$ (Table V). In the $\mathrm{SAD}$ group, no significant differences were found between the two countries in terms of numbers of patients presenting with increased minimization/denial.

We found psychometric adequacy for the MD subscale and for 4 of the 5 other CTQ subscales. The internal consistency (Cronbach alpha) of the 5th subscale of physical neglect was relatively low $(0.41)$.

\section{Discussion}

The influence of childhood trauma as a specific environmental factor on the development of adult psychopathology is far from being elucidated. The present study is part of a collaborative project between research groups from South Africa and Sweden focusing on genetic and environmental factors contributing to anxiety disorders. As such, our study contributes to the literature on anxiety disorder and early life adversity by investigating whether childhood trauma history is predictive of adult SAD or PD in South African and Swedish patients.

Our findings suggest that patients from South Africa reported significantly higher trauma rates, and sexual abuse, and emotional and physical neglect in particular, than Swedish patients. In the combined analyses, patients with $\mathrm{SAD}$ reported higher rates of childhood emotional abuse compared to those with PD; childhood emotional abuse, more specifically, show a predictive association with SAD vs. PD in adulthood. In addition, our findings also suggest that the psychometric qualities of the CTQ subscales including the MD subscale were adequate with the exception of the physical neglect subscale with its relatively low internal consistency.

\begin{tabular}{|c|c|c|c|}
\hline Diagnosis & South Africa & Sweden & P-Value \\
\hline $\begin{array}{l}\text { Panic Disorder } \\
\text { Social Anxiety Disorder } \\
\text { P-Value }\end{array}$ & $\begin{array}{l}9.81(4.94) \\
11.77(5.66) \\
0.4\end{array}$ & $\begin{array}{l}8.65(4.0) \\
10.13(4.92) \\
0.53\end{array}$ & \begin{tabular}{|l|}
1.0 \\
0.39
\end{tabular} \\
\hline
\end{tabular}

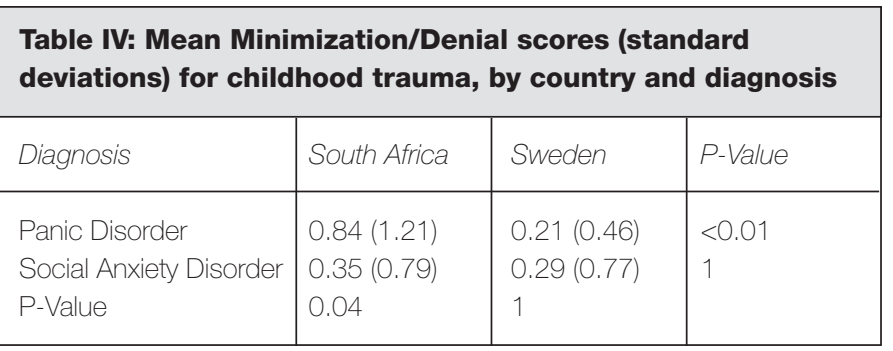

Figure 2: Bar graph depicting the significant interaction between diagnosis and country for Minimization / Denial

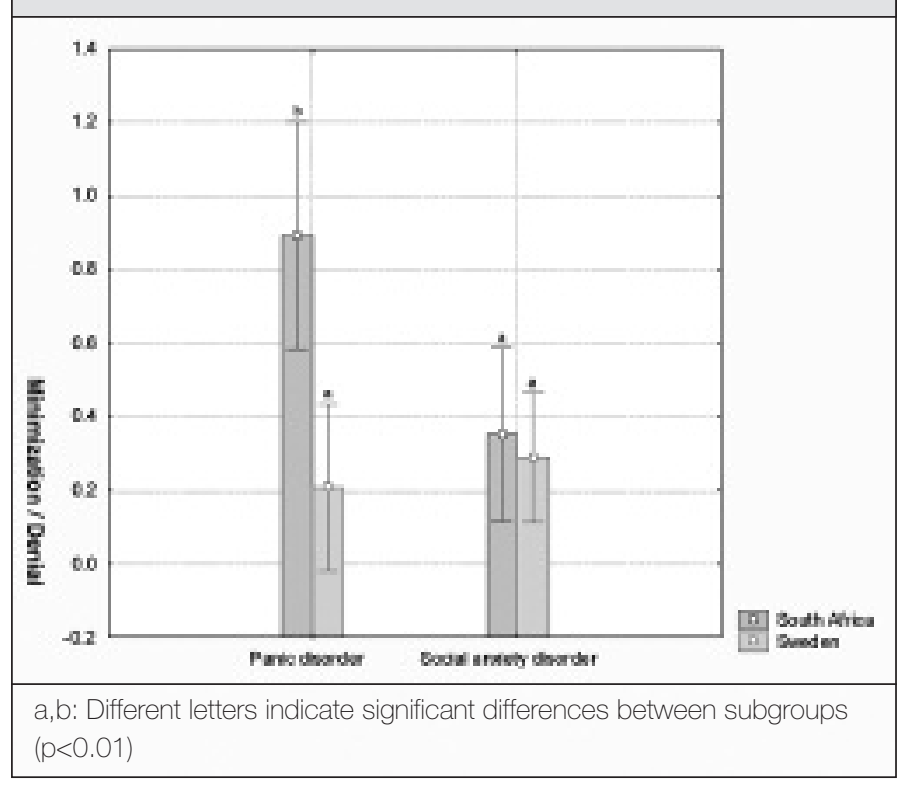

Of note, our findings also suggest that South African patients with PD had more minimization/denial than Swedish PD patients, and that more of the South African patients with PD possibly underreported maltreatment compared to their Swedish counterparts. However, no such country differences were found among SAD patients. In addition, female patients with SAD/PD reported higher rates of emotional abuse and sexual abuse than males.

Our finding that South Africans had higher rates of recalled

Table V: Minimization/Denial of childhood trauma, by country and diagnosis

\begin{tabular}{|c|c|c|c|c|}
\hline Diagnosis & Md Dichotomy & South Africa & Sweden & Statistics \\
\hline Panic Disorder & $\begin{array}{l}\text { Md Total Score }<1 \\
\text { Md Total Score }>=1\end{array}$ & $\begin{array}{l}23(62.16 \%) \\
14(37.84 \%)\end{array}$ & $\begin{array}{l}42(81 \%) \\
10(19 \%)\end{array}$ & $\chi^{2}=3.765, P=0.052$ \\
\hline Social Anxiety Disorder & $\begin{array}{l}\text { Md Total Score }<1 \\
\text { Md Total Score }>=1\end{array}$ & $\begin{array}{l}38(79.17 \%) \\
10(20.83 \%)\end{array}$ & $\begin{array}{l}71(86 \%) \\
12(14 \%)\end{array}$ & $\chi^{2}=0.866, P=352$ \\
\hline
\end{tabular}


childhood trauma compared to Swedish patients was expected. Compared to Sweden (a high-income developed country),

South Africa, a lower-middle income country, is characterized by high rates of crime, violence and poverty. It has been suggested that if the environment - which in South Africa is characterized by "... a long history of abuse of human rights, repression, violence, alcohol related problems, malnutrition, and poverty"24 is a major determinant of mental health, then the mental health of South Africans might be expected to be poorer than that of people elsewhere. ${ }^{24}$ In addition, a large body of evidence confirms that many people in the developing world go without health care from which they could benefit greatly, rendering them more vulnerable to mental disorders than citizens from developed countries like Sweden which is known for its easily accessible, "top-rate" and comprehensive (mental) health system. ${ }^{25,26}$ However, it is important to note that not all South African participants have had exposure to the aforementioned adversities. Similarly, participants from Stockholm may have also been exposed to some of these negative circumstances, even though they come from a first-world context.

More studies on the extent and nature of the influence of adverse factors on the development of mental disorders, and the anxiety disorders in particular, in different environments, are needed. Findings from an unpublished study in a subset of the current South African sample (Lochner, Seedat \& Stein: The role of childhood trauma across anxiety disorders: Obsessivecompulsive, social anxiety \& panic disorder. Unpublished data 2009) which compared anxiety disorder patients with healthy controls (recruited from the same South African communities) in terms of trauma history, demonstrated that patients with obsessive-compulsive disorder, SAD or PD reported significantly more childhood trauma in general, and more emotional neglect and emotional abuse in particular, than healthy controls, suggesting a possible role for trauma in the development of these disorders. In Sweden, a recent study comparing SAD patients and controls in terms of the frequency and impact of life events across different periods of life, also suggested a possible role for negative interpersonal events in the development of SAD. ${ }^{27}$

Analyses of the combined sample as well as data from South Africa and Sweden separately, suggest that emotional abuse might be predictive of SAD vs. PD in later life. Some other studies have suggested a relation between childhood emotional abuse history and the presence of anxiety disorders, particularly $\mathrm{SAD}$, in adulthood. ${ }^{8}$ Also consistent with our findings, a recent report of CTQ results in 104 subjects (the majority being men) with $\mathrm{SAD}$ found an increased total trauma score, particularly due to emotional abuse and neglect. ${ }^{28}$ A specific and strong link between emotional abuse and anxiety disorders in adulthood was confirmed in a recent study that predicted that reports of childhood emotional abuse would exhibit a specific relationship with depressive and anxiety disorders among adult psychiatric outpatients. Self-reported histories of teasing or bullying experiences (which can be construed as emotional abuse) have also been found to play a role in the development of SAD specifically ${ }^{29}$ - a finding that also highlights the link between perceptions of teasing in childhood and the development of SAD.

Although rates of emotional abuse among PD patients were lower compared to that of SAD patients, an association between emotional abuse and PD has also been suggested in previous work. For example, almost 10\% of PD patients attending an
Anxiety Disorders Program at the University of Michigan, reported childhood emotional abuse. ${ }^{13}$ In another study that compared the level of emotional, physical and sexual childhood abuse as well as physical and emotional childhood neglect between 40 adult patients with PD and 40 healthy control subjects, it was found that the patients had significantly higher levels of physical and emotional abuse. ${ }^{14}$

It should be borne in mind that recollection of childhood traumas may be impaired by the disorder under study; trauma may be over-reported in the search for a meaningful explanation of disorder aetiology, or it may be underreported due to intentional or unintentional memory suppression/denial since many studies investigating childhood trauma report high rates of MD scores. ${ }^{30}$ As most studies ${ }^{30}$ investigating childhood trauma report high rates of $\mathrm{MD}$, the high rate of minimization in our sample was not unexpected. MD scores differed significantly between the 2 sites, with higher rates generally in the South African group. These higher MD rates in the South Africa sample were particularly evident in patients with $\mathrm{PD}$, and may suggest that the higher rates of childhood trauma reported by these patients may, in fact, be an under-representation of actual childhood adverse experiences. Since minimization or denial of childhood trauma may have biased our findings, we repeated the regression analyses with exclusion of patients with extreme MD scores (i.e. a MD total score of 3). However, our findings from the regression analyses were not significantly altered after exclusion of data from patients with a MD score of 3. Arguably, in some instances minimization or denial may facilitate coping with trauma, and by implication influence recovery and possible manifestation of psychopathology. More work on the 'adaptive' role of minimization and denial in the context of childhood trauma is warranted. In addition, it should be borne in mind that various types of adverse events may in some cases not be independent of each other. For example, when either physical or sexual abuse is present, emotional abuse is likely to also be present.

Our findings overall support previous findings of the psychometric adequacy of most of the CTQ subscales. An internal consistency reliability coefficient of 0.70 or more (obtained on a substantial sample) is usually required. ${ }^{31}$ Streiner and Normann suggested that alpha of 0.70 or more should be regarded as satisfactory, and that 0.50 or more is acceptable for group comparisons. ${ }^{32}$ The Cronbach alpha of the 5th subscale assessing physical neglect was, however, below both these cutoffs, suggesting that the items comprising this subscale may be multidimensional or not highly correlated with one another. Interestingly, there seems to be agreement across many CTQ studies that the physical neglect subscale has limited reliability, is not homogenous, and that its items may be variably interpreted ${ }^{33}$ i.e. characteristics that may have biased our findings to some extent. This possible limitation should be considered in future studies of childhood interpersonal trauma using the CTQ. In addition, some of the South African participants were referred by their clinicians for participation but the majority of the sample was volunteers recruited from the community via the media compared to all of the Swedish participants who were volunteers. Although the different recruitment methods may have resulted in differing subsets of patients, it is unlikely that it would have had significant bearing on the endorsement of childhood adversity, as participants were primarily recruited on the presence/absence of an anxiety 
disorder diagnosis. Another limitation of this cross-sectional study is that it was retrospective in nature and thus childhood trauma, in particular, may have been subject to recall bias. In addition, given the substantial comorbidity of depressive, substance abuse and anxiety disorders, the fact that we did not control for these possibly comorbid disorders in our analyses, could possibly be construed as a limitation. Future studies on the association of the CTQ in patients with pure versus comorbid depressive and anxiety disorder diagnoses are needed.

\section{Conclusion}

Longitudinal studies should focus on the potential sequential development of SAD and PD among individuals with a history of childhood emotional abuse. Our findings, together with those of other studies on trauma and anxiety, suggest that specific forms of childhood trauma as well as other possible risk factors such as gender, historical context/culture and biological vulnerability should be considered when assessing risk for anxiety disorder morbidity in adulthood. This exploratory study contributes to the ongoing search for antecedent aetiological factors for anxiety disorders.

\section{Acknowledgements}

The Medical Research Council of South Africa, the National Research Fund of South Africa, and the Swedish International Development Agency and Science Council grant \#348-20045668 supported this work.

\section{References}

1. Brown GW, Harris TO, Eales MJ. Social factors and comorbidity of depressive and anxiety disorders. Br J Psychiatry Suppl 1996;30:50-57.

2. Heider D, Matschinger H, Bernert S, Alonso J, Brugha TS, Bruffaerts R et al. Adverse parenting as a risk factor in the occurrence of anxiety disorders a study in six European countries. Soc Psychiatry Psychiatr Epidemiol 2008; 43(4):266-272

3. Cicchetti D, Toth SL, Maughan A. An ecological-transactional model of child maltreatment. In: Sameroff AJ, Lewis M, Miller SM, editors. Handbook of developmental psychopathology. New York: luwer Academic/Plenum Press, 2000: 689-722.

4. David D, Giron A, Mellman TA. Panic-phobic patients and developmental trauma. J Clin Psychiatry 1995; 56(3):113-117.

5. Bandelow B, Charimo TA, Wedekind D, Broocks A, Hajak G, Ruther E. Early traumatic life events, parental rearing styles, family history of mental disorders, and birth risk factors in patients with social anxiety disorder. Eur Arch Psychiatry Clin Neurosci 2004; 254(6):397-405.

6. Bandelow B, Spath C, Tichauer GA, Broocks A, Hajak G, Ruther E. Early traumatic life events, parental attitudes, family history, and birth risk factors in patients with panic disorder. Compr Psychiatry 2002; 43(4):269-278.

7. Marteinsdottir I, Tillfors M, Furmark T, Anderberg UM, Ekselius L. Personality dimensions measured by the Temperament and Character Inventory (TCI) in subjects with social phobia. Nord J Psychiatry 2003; 57(1):29-35.

8. Gibb BE, Chelminski I, Zimmerman M. Childhood emotional, physical, and sexual abuse, and diagnoses of depressive and anxiety disorders in adult psychiatric outpatients. Depress Anxiety 2007; 24(4):256-263.

9. Asher SR, Coie JD. Peer rejection in childhood. In: Asher SR, Coie JD, editors. New York: Cambridge University Press, 1990.

10. Hackmann A, Surawy C, Clark DM. Seeing yourself through others' eyes: $A$ study of spontaneously occurring images in social phobia. Behavioural and Cognitive Psychotherapy 1998; 26(1):3-12.

11. La Greca AM, Silverman WK, Wasserstein SB. Children's predisaster functioning as a predictor of posttraumatic stress following Hurricane Andrew. J Consult Clin Psychol 1998; 66(6):883-892.

12. Slee PT. Situational and interpersonal correlates of anxiety associated with peer victimisation. Child Psychiatry Hum Dev 1994; 25(2):97-107.

13. Young EA, Abelson JL, Curtis GC, Nesse RM. Childhood adversity and vulnerability to mood and anxiety disorders. Depress Anxiety 1997; 5(2):66-72

14. Bonevski D, Novotni A. [Child abuse in panic disorder]. Med Pregl 2008; 61(3-4):169-172.

15. Chartier MJ, Walker JR, Stein MB. Social phobia and potential childhood risk factors in a community sample. Psychol Med 2001; 31 (2):307-315.

16. Stein MB, Walker JR, Anderson G, Hazen AL, Ross CA, Eldridge G et al. Childhood physical and sexual abuse in patients with anxiety disorders and in a community sample. Am J Psychiatry 1996; 153(2):275-277.

17. Safren SA, Gershuny BS, Marzol P, Otto MW, Pollack MH. History of childhood abuse in panic disorder, social phobia, and generalized anxiety disorder. J Nerv Ment Dis 2002; 190(7):453-456.

18. Mancini C, Van Ameringen M, MacMillan H. Relationship of childhood sexual and physical abuse to anxiety disorders. J Nerv Ment Dis 1995; 183(5):309-314.

19. Hofmann SG, Levitt JT, Hoffman EC, Greene K, Litz BT, Barlow DH. Potentially traumatizing events in panic disorder and other anxiety disorders. Depress Anxiety 2001; 13(2):101-102.

20. Bernstein DP, Fink L, Handelsman L, Foote J, Lovejoy M, Wentzel Ket al. Initial reliability and validity of a new retrospective measure of child abuse and neglect. Am J Psychiatry 1994; 151:1132-1136.

21. First MB, Spitzer RL, Gobbon M, Williams JBW. Structured clinical interview for DSM-IV Axis I disorders - Patient edition (SCID-I/P, Version 2.0, 8/98 revision). New York: New York State Psychiatric Institute, Biometrics Research Department, 1998.

22. Sheehan DV, Lecrubier Y, Sheehan KH, Amorim P, Janavs J, Weiller E et al. The Mini-International Neuropsychiatric Interview (M.I.N.I.): The development and validation of a structured diagnostic psychiatric interview for DSM-IV and ICD-10. J Clin Psychiatry 1998; 59 Suppl 20:22-33.

23. Bernstein D, Fink L. Manual for the Childhood Trauma Questionnaire. New York: The Psychological Cooperation, 1998.

24. Kale R. New South Africa's mental health. BMJ 1995; 310(6989):1254-1256.

25. Triggle N. http://news.bbc.co.uk/go/pr/fr/-/2/hi/health/4460098.stm. BBC News . 2005. Ref Type: Electronic Citation

26. Silfverhielm H, Kamis-Gould E. The Swedish mental health system. Past, present, and future. Int J Law Psychiatry 2000; 23(3-4):293-307.

27. Marteinsdottir I, Svensson A, Svedberg M, Anderberg UM, von Knorring L. The role of life events in social phobia. Nord J Psychiatry 2007; 61:201-212.

28. Childhood maltreatment linked to greater symptom severity and poorer quality of life and function in social anxiety disorder. 90 Mar; Albuquerque: Annual Conference of the Anxiety Disorders Association of America (ADAA), 2009.

29. MCCabe RE, Antony MM, Summerfeldt LJ, LisS A, Swinson RP. Preliminary examination of the relationship between anxiety disorders in adults and self-reported history of teasing or bullying experiences. Cogn Behav Ther 2003; 32(4):187-193.

30. Villano CL, Cleland C, Rosenblum A, Fong C, Nuttbrock L, Marthol M et al. Psychometric Utility of the Childhood Trauma Questionnaire with Female Street-Based Sex Workers. J Trauma Dissociation 2004; 5(3):33-41.

31. Nunnally J. Psychometric Theory. New York: McGraw-Hill, 1978.

32. Streiner DL, Normann GR. Health measurement scales: a practical guide to their development and use. Oxford: Oxford University Press, 1989.

33. Gerdner A, Allgulander C. Psychometric properties of the Swedish version of the Childhood Trauma Questionnaire - Short Form (CTQ-SF). Nordic Journal of Psychiatry 2009; 63(2):160-170 